\title{
CXCR4, un récepteur aux multiples fonctions
}

Le récepteur CXCR4 appartient à la grande famille des récepteurs à sept domaines transmembranaires couplés aux protéines $\mathrm{G}$ et, plus précisément, à la famille des récepteurs des chimiokines. Découvert en 1994 après le clonage de son $\mathrm{ADNc}$ et classé dans le groupe des récepteurs orphelins, parce que sans ligand connu, le récepteur CXCR4 fit la une de l'actualité scientifique en mai 1996 lorsque l'équipe de Berger (Bethesda, MD, USA) [1] découvrit que ce récepteur CXCR4 servait de porte d'entrée au virus de l'immunodéficience humaine (VIH) $(\mathrm{m} / \mathrm{s} 1996$, $n^{\circ} 10, p$. 1185). En s'associant à la molécule CD4, CXCR4 joue le rôle de co-facteur, permettant aux souches T-tropiques du virus, c'est-àdire infectant les lymphocytes $\mathrm{T}$, de pénétrer dans la cellule hôte.

Le ligand naturel de CXCR4 fut découvert peu de temps après $[2,3]$. Il s'agit d'une chimiokine appelée SDF-1, pour stromal cell-derived factor-1, car elle a été initialement clonée à partir d'une bibliothèque d'ADNc issus de cellules stromales de la moelle osseuse. Au contraire des autres chimiokines dont l'expression est induite, SDF-1 est synthétisée de façon constitutive par ces cellules stromales. In vitro, la prolifération des cellules souches des lymphocytes B est activée par SDF-1 [4]. En 1996, l'étude du rôle physiologique de SDF-1 fut entreprise par l'invalidation, chez la souris, du gène codant pour cette molécule. Nagasawa et al. (Osaka, Japon) découvraient que les souris $S D F-1^{-1-}$ mouraient précocement, in utero, dans la grande majorité des cas [5]. Les embryons $S D F$ $1^{-/}$présentaient un nombre réduit de cellules souches des lymphocytes $\mathrm{B}$, aussi bien dans le foie foetal que dans la moelle osseuse, tandis que la quantité des cellules souches myéloïdes n'était réduite que dans la moelle osseuse. Ces résultats suggéraient que SDF-1 était responsable à la fois de la lymphopoïèse $B$ et de la myélopoï̀se. De plus, les quelques souriceaux qui naissaient mouraient dans la première heure d'insuffisance cardiaque, présentant un défaut dans la formation du septum ventriculaire du cœur.

La confirmation du rôle physiologique joué par le couple SDF1/CXCR4 vient d'être apportée dans deux articles récents publiés dans la revue Nature [6, 7]. En effet, l'étude du rôle physiologique de CXCR4 a été entreprise de nouveau par la technique d'invalidation de son gène chez la souris. Les travaux publiés par Tachinaba et al. (Osaka, Japon) [6] montrent que CXCR4 est exprimé dans les cellules endothéliales des vaisseaux sanguins et que les souris CXCR4 $4^{--}$présentent des anomalies de l'hématopoïèse et de la formation du cœur, identiques à celles observées par Nagasawa et al. avec les souris $S D F-1^{-1-}[5]$. Cela démontre de façon claire la complémentarité du couple SDF-1/CXCR4, sans redondance d'activité de la part du ligand ou du récepteur. En outre, les souris $C X C R 4^{-/-}$ont des défauts dans le développement vasculaire et, plus particulièrement, dans la vascularisation du tractus gastro-intestinal, suggérant un nouveau rôle du couple SDF-1/CXCR4 dans la vascularisation des organes. Zou et al. (New York, USA) [7] montrent eux aussi le rôle majeur joué par CXCR4 dans l'hématopoïèse et dans l'organogenèse cardiaque. L'étude plus approfondie du système nerveux central et du cerveau des souris $C X C R 4^{-/}$révèle une anomalie du développement cérébral chez les foetus. Les cerveaux des souris mutées contiennent plusieurs cellules de la couche granuleuse externe à des endroits inhabituels du cerveau, suggérant que la migration de ce type cellulaire ne s'est pas bien effectuée chez les souris $\mathrm{CXCR}^{-/}$. Ces travaux sont la première démonstration de l'implication d'un récep- teur des chimiokines dans la migration des cellules du cerveau et dans la mise en place du système nerveux central. Ces études peuvent également servir de tremplin à la compréhension de l'infection du cerveau par le VIH car il est bien connu que les malades du SIDA dans la phase terminale de leur maladie peuvent être atteints de démence.

L'ensemble de ces études montre que le rôle des chimiokines et de leurs récepteurs ne se limite pas seulement à l'attraction des leucocytes sur le site inflammatoire, mais que les chimiokines et leurs récepteurs sont impliqués dans un bon nombre de mécanismes de communications cellulaires. SDF-1 et CXCR4 sont peut-être les deux premières molécules d'une longue série impliquées dans la migration des cellules lors de la mise en place des organes au cours du développement embryonnaire.

M.S.

1. Feng Y, Broder CC, Kennedy PE, Berger EA. HIV-1 entry cofactor : functional cDNA cloning of a seven-transmembrane, $\mathrm{G}$ protein-coupled receptor. Science 1996; 272: 872-7.

2. Bleul CC, Farzan M, Choe H, Parolin C, ClarkLewis I, Sodroski J, Springer TA. The lymphocyte chemoattractant SDF-1 is a ligand for LESTR/fusin and blocks HIV-1 entry. Nature 1996; 382: 829-33.

3. Oberlin E, Amara A, Bachelerie F, Bessia C, Virelizier JL, et al. The CXC chemokine SDF-1 is the ligand for LESTR/fusin and prevents infection by T-cell-line-adapted HIV-1. Nature 1996; 382: 833-5.

4. Nagasawa T, Kikutani H, Kishimoto T. Molecular cloning and structure of a pre-B-cell growth-stimulating factor. Proc Natl Acad Sci USA 1994; 91 : 2305-9.

5. Nagasawa T, Hirota S, Tachibana K, Takakura $\mathrm{N}$, Nishikawa S, et al. Defects of B-cell lymphopoiesis and bone-marrow myelopoiesis in mice lacking the CXC chemokine PBSF/SDF-1. Nature 1996; 382: 635-8.

6. Tachibana K, Hirota S, Iizasa H, Yoshida H, Kawabata K, et al. The chemokine receptor CXCR4 is essential for vascularization of the gastrointestinal tract. Nature 1998; 393: 591-5.

7. Zou YR, Kottmann AH, Kuroda M, Taniuchi I, Littman DR. Function of the chemokine receptor CXCR4 in haematopoiesis and in cerebellar development. Nature 1998; 393: 595-9. 